\title{
Estimation of Cholesterol and Bile Acid Turnover in Man by Kinetic Analysis
}

\author{
Steven H. Quarfordt and Mary F. Greenfield \\ From the Department of Medicine, Duke University Medical Center, and \\ Cooperative Lipid Laboratory, Veterans Administration Hospital, \\ Durham, North Carolina 27705
}

\begin{abstract}
A в S T R A C T The kinetics of cholesterol and bile acid turnover were determined from an analysis of the biliary lipids after a single intravenous injection of labeled cholesterol. A compartmental model was designed for the system, and the fractional metabolic rates and fluxes were determined in one lean and two obese normal humans. Each of the normals converted about $3 \%$ per day of their rapidly miscible cholesterol pool to cholic acid and $1 \%$ per day or less to chenodeoxycholate. Cholate was catabolized at about twice the rate of the dihydroxy bile acids in these normals. Two of the normals were fed corn oil with little change in their kinetic parameters from the control state. The other normal received cholestyramine and dramatically increased the bile acid flux with little change in neutral sterol catabolism. A cirrhotic patient was also studied by this technique and noted to have kinetic parameters quite different from the normals.
\end{abstract}

\section{INTRODUCTION}

Human cholesterol turnover has been evaluated by a number of techniques (1-8). Some studies assayed the fecal products of cholesterol catabolism and estimated the flux of both neutral and acidic sterols (1). The uncertainties of fecal flow and bacterial sterol metabolism made fecal data difficult to interpret and led to the subsequent use of markers for these variables (2-4). Other studies (5-8) used plasma cholesterol radioactivity decay data to determine cholesterol turnover. Initially a single compartment model was assumed to represent the body cholesterol system (5). Recent studies (6-8) have used a twocompartment model to simulate the biexponential plasma cholesterol decay curve. To calculate parameters of cholesterol kinetics in the two-compartment system it was assumed that all of the cholesterol catabolism oc-

Received for publication 5 January 1973 and in revised form 7 March 1973. curred in the rapidly miscible pool (RMP) ${ }^{1}$ of which the plasma cholesterol was part. This type of analysis did not determine the fraction of cholesterol catabolism that occurred as neutral and as acidic sterol.

The present study describes a compartmental analysis of cholesterol and bile acid kinetics in a lean normal subject, two obese subjects, and a patient with macronodular cirrhosis. After an intravenous injection of radioactive cholesterol, the specific activity time course of plasma cholesterol and biliary neutral and acidic sterols was followed for periods up to 3 mo. This approach permitted the calculation of the amount of cholesterol catabolism that occurred as neutral sterol and as bile acid and provided an opportunity to document the assumption that RMP cholesterol is the predominant precursor of the primary bile acids. The normal subjects were studied both in their control state and again after their plasma cholesterol concentrations were reduced by either cholestyramine or corn oil feedings.

\section{METHODS}

Subjects. The normal subjects were either laboratory personnel or graduate students with negative medical histories and physical examinations. Their clinical characteristics and plasma cholesterol and triglyceride concentrations are described in Table I. All subjects were studied as outpatients, and their cholesterol intakes and diets were monitored intermittently. The subjects' weights did not vary more than $2 \mathrm{~kg}$, and each day they took in between 500 and $600 \mathrm{mg}$ of cholesterol during the period of study. Two of the subjects were placed on a high corn oil diet after their initial study. Their saturated fat intake was reduced so that they consumed an isocaloric diet with a polyunsaturated to saturated ratio of about $1.7 / 1$ that contained about $400 \mathrm{mg}$ cholesterol daily. Another subject remained on the same diet and was given $16 \mathrm{~g}$ of cholestyramine each day after the initial study was completed. When new steady-

${ }^{1}$ Abbreviations used in this paper: RMP, rapidly miscible cholesterol pool or compartment 1; SMP, slowly miscible cholesterol pool or compartment 2 . 
TABLE I

Clinical Characteristics of Subjects

\begin{tabular}{|c|c|c|c|c|c|c|c|}
\hline \multirow[b]{2}{*}{ Subject } & \multirow[b]{2}{*}{ Age } & \multirow[b]{2}{*}{ Sex } & \multirow[b]{2}{*}{ Weight } & \multirow[b]{2}{*}{ Clinical diagnosis } & \multirow{2}{*}{$\begin{array}{l}\text { Triglyceride } \\
\text { concentrations }\end{array}$} & \multicolumn{2}{|c|}{ Cholesterol concentrations } \\
\hline & & & & & & Control & Experimental* \\
\hline & $y r$ & & $k g$ & & $m g / d l$ & \multicolumn{2}{|c|}{$m g / d l$} \\
\hline R. S. & 22 & $\mathrm{M}$ & 65 & Normal & 82 & $167 \pm 3 \ddagger$ & $140 \pm 4$ \\
\hline E. Y. & 45 & $\mathrm{~F}$ & 96 & Obese & 64 & $166 \pm 3$ & $134 \pm 3$ \\
\hline O. H. & 47 & $\mathrm{~F}$ & 100 & Obese & 94 & $156 \pm 3$ & $117 \pm 5$ \\
\hline D. T. & 62 & $\mathbf{M}$ & 60 & Macronodular cirrhosis & 136 & $426 \pm 5$ & \\
\hline
\end{tabular}

* Subjects R. S. and E. Y. were placed on the corn oil regimen described in the text during the experimental period. Subject D. H. was placed on $16 \mathrm{~g}$ of cholestyramine during this period.

$\ddagger$ Mean \pm standard error of the mean.

state plasma concentrations were stable usually after about 1 mo of corn oil or Cholestyramine feeding, a second study was begun. Plasma cholesterol concentrations were determined biweekly prior to the start of each study to be certain that steady-state conditions existed. Studies were begun if after 2 wk the coefficient of variation for plasma cholesterol was less than $8 \%$ and no concentration trend was apparent.

Procedure. Human plasma was labeled by incubating it with an acetone dispersion of $\left[1-\alpha-{ }^{3} \mathrm{H}\right]$ cholesterol (Amersham/Searle Corp., Arlington Heights, Ill.) for $2 \mathrm{~h}$ at $37^{\circ} \mathrm{C}$ similar to the method of Goodman and Noble (6). After standing for $6 \mathrm{~h}$ at room temperature the mixture was filtered through a $0.22 \mu \mathrm{m}$ Millipore filter into a sterile container. The specific activity of free cholesterol was the same for the very low, low, and high density lipoproteins, and less than $6 \%$ of the activity was in cholesteryl esters. On the initial day of the study each subject was injected intravenously with between $25-30 \mu \mathrm{Ci}$ of the cholesterollabeled plasma. Blood samples were obtained from the antecubital veins and collected in tubes containing disodium EDTA $(1 \mathrm{mg} / \mathrm{ml}$ blood). Bile samples were obtained by duodenal intubation using a Dreiling tube (9) to aspirate gastric contents through one lumen and duodenal contents through the other. Bile enrichment of the duodenal fluid was produced by an intravenous injection of $75 \mathrm{dog}$ units of Cholecystokinin (Karolinska, Stockholm) in $50 \mathrm{ml}$ of normal saline. A small aliquot (equivalent to about $2.5 \mathrm{mg}$ of cholesterol and $15 \mathrm{mg}$ of total bile acid) of the duodenal fluid was retained for analysis, and the rest was returned to the duodenum. Bile and blood samples were always obtained after an overnight fast of about $14 \mathrm{~h}$. For the initial week of the study samples were usually obtained every day and on subsequent weeks less frequently to a minimum of one determination biweekly. Studies were usually continued for periods of $10-11 \mathrm{wk}$.

At the conclusion of the study the subjects received an intravenous injection of from 2 to $3.5 \mu \mathrm{Ci}$ of either [24$\left.{ }^{14} \mathrm{C}\right]$ sodium glycocholate or unconjugated $\left[24-{ }^{14} \mathrm{C}\right]$ cholic acid (Tracerlab, Waltham, Mass.) dispersed in $10 \mathrm{ml}$ of buffered saline. By thin-layer and gas-liquid chromatography these were noted to be greater than $90 \%$ pure. Bile samples were obtained for 6 days after the labeled cholate injection. Small aliquots were retained for determinations of the specific activity time course of total biliary cholate.

Chemistry. Plasma was obtained from blood by centrifuging at $2,500 \mathrm{rpm}$ for $25 \mathrm{~min}$. Total cholesterols were determined on duplicate aliquots of plasma by the proce- dure of Abell, Levy, Brodie, and Kendall (10). Aliquots of the ligroin phase were taken for mass and radioactivity determinations. Free cholestenol-specific activities were also determined on early plasma samples in some of the studies. The plasmas were extracted by the Dole procedure (11), and the petroleum ether phase was removed, dried under nitrogen, and taken up in small amounts of $1 / 1$ chloroform methanol for thin-layer chromatography. The samples were applied to a $250 \mu \mathrm{m}$ silica gel $\mathrm{G}$ plate and developed in an 80:20:1 petroleum ether-diethyl etheracetic acid system. The isolated free sterol was eluted with $1 / 1$ chloroform-methanol, and aliquots were assayed for radioactivity and for mass by the method of Abell et al. (10).

Neutral sterols and bile acids were removed from the bile by a minor modification of the methods described by Miettinen, Ahrens, and Grundy $(12,13)$. The duodenal fluid was gently refluxed at $85^{\circ} \mathrm{C}$ for $1 \mathrm{~h}$ with $1 \mathrm{~N} \mathrm{NaOH}$ in $90 \%$ ethanol. The hydrolysate was partitioned between water and petroleum ether. The petroleum ether phase containing the neutral sterols was removed and washed with $1 \mathrm{~N} \mathrm{NaOH}$ in $50 \%$ ethanol. The petroleum ether was dried and made to volume, and aliquots were removed for assay of radioactivity and determination of mass by the method of Abell et al. (10).

$4 \mathrm{ml}$ of $10 \mathrm{~N} \mathrm{NaOH}$ was added to the aqueous phase and it was saponified in an autoclave at $120^{\circ} \mathrm{C}$ and $15 \mathrm{lb} /$ in $^{2}$ for $3 \mathrm{~h}$. The saponified mixture was acidified to $\mathrm{pH} 2$ with concentrated $\mathrm{HCl}$, and the bile acids were extracted with chloroform-methanol $2: 1$ and then with chloroform. The pooled extracts were dried under nitrogen, taken up in $1 / 1$ chloroform-methanol, and plated on $1 \mathrm{~mm}$ silica gel $\mathrm{G}$ plates which were then developed in a $1: 1: 0.2$ isooctane, ethyl acetate, and acetic acid system. The trihydroxy and dihydroxy bile acid bands were identified after exposure to $\mathrm{I}_{2}$ vapor by their co-migration with known standards. The bile acids were eluted from the gel with three washes of absolute ethanol. The dihydroxy bile acid band was dried under nitrogen and replated on a $350 \mu \mathrm{m}$ silica gel $\mathrm{G}$ plate and developed for $3 \mathrm{~h}$ in the same solvent system along with chenodeoxycholic and deoxycholic acid standards. A good separation of the two bile acids was achieved, and the bands were eluted from the gel with absolute ethanol. All the bile acids were dried and made to volume, and aliquots were obtained for assay of radioactivity and for determination of mass by the steroid dehydrogenase technique (14).

The bile acids isolated by thin-layer chromatography were checked for purity by gas-liquid chromatography (15). 
Bile acids were methylated and trifluoroacetylated prior to running on a $Q F-1$ column at $270^{\circ} \mathrm{C}$ using $5 \beta$-cholanic acid as an internal standard. No contamination of the isolated bile acids was appreciated in any of the samples evaluated. Bile acid radioactivity was assayed in a scintillation system using dioxane, anisole, dimethyloxyethane, as solvents containing $0.7 \%$ diphenyloxazole and $0.1 \% \quad 1,4$ bis-diphenyloxazolyl benzene. Cholesterol radioactivity was assayed in a $0.5 \%$ diphenyloxazole in toluene scintillation system. All samples were counted in a Packard Model 3375 liquid scintillation spectometer, and quenching was determined by an internal standard technique. The amount of radioactivity injected into each subject was determined by knowing the radioactivity and density of the filtered plasma that was injected and carefully weighing the syringe when full and after the injection. Specific activities were expressed as fraction of injected dose, after corrections for counting efficiency, storage decay, and for the small differences between the molecular weights of cholesterol and the bile acids.

Calculations. The decay of the biliary $\left[{ }^{14} \mathrm{C}\right]$ cholic acid specific activity was evaluated by a linear regression program on an IBM 1130 computer to obtain an estimate of the cholate pool size and its variance. The pool sizes of chenodeoxycholate and deoxycholate were estimated from the cholic acid pool size determination and from the mean proportions of the three major bile acids found in the duodenum, after Cholecystokinin stimulation. It was necessary to use these estimates of the biliary pool sizes to assure a rapid convergence of the kinetic problem to a solution.

The model selected for the evaluations of the $\left[{ }^{3} \mathrm{H}\right]$ cholesterol and bile acid data is described in Fig. 1. This model combines the two-compartment model previously used for cholesterol kinetics (6-8), with a model consistent with known bile acid metabolism. The simulation analysis and modeling (SAAM 23) program of Berman and Weiss (16) was adapted to an IBM $370-165$ computer and used to determine the fractional rate constants of the system and their variance. Details of this technique are explicitly provided in the manual for either the SAAM 23 or 25 programs (16). It was necessary to provide good initial estimates of the biliary pool sizes and to limit their variability in order to converge to a solution. Calculations of steady-state pool sizes and transport rates were also determined.

\section{RESULTS}

The model was initially constructed with a two-pool cholesterol system, having primary bile acid inputs from both the RPM and slowly miscible cholesterol pool (SMP). The bile acid input from the SMP was determined to be a very small value, approximately zero, in the solution of each study. The experimental primary bile acid specific activity functions were simulated very well with only an input from the RMP. For these reasons the conversion of SMP cholesterol to cholic and chenodeoxycholic acid was deleted, and the model used for the studies (Fig. 1) had biliary inputs from only the RMP.

Systematic deviations in the early time course of bilefree cholesterol specific activity from that of total plasma cholesterol were noted in all studies. The biliary neutral

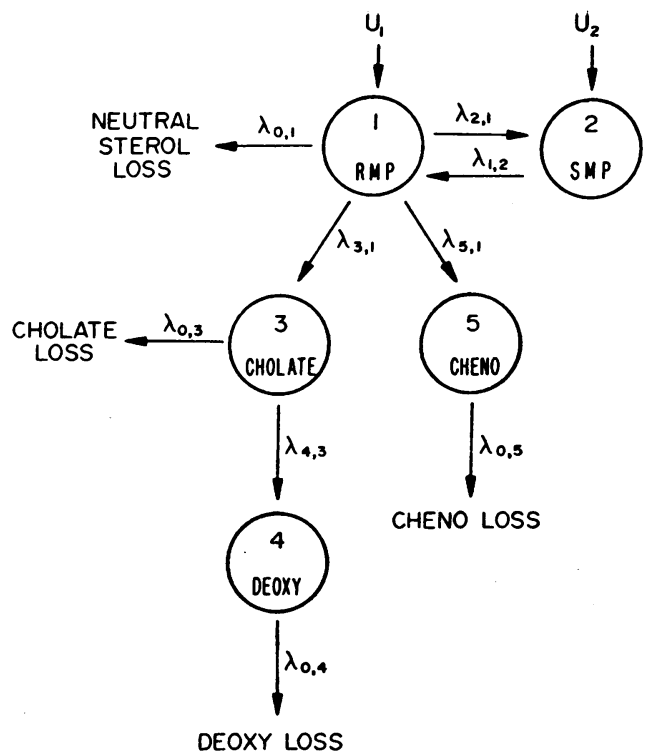

Figure 1 The multicompartmental model used for the analysis of cholesterol kinetics. Each of the five compartments contain an abbreviation indicating what is represented. Compartment 1, rapidly miscible cholesterol pool (RMP) ; compartment 2, slowly miscible cholesterol pool (SMP) ; compartment 3, cholic acid pool; compartment 4, deoxycholic acid pool; compartment 5, chenodeoxycholic acid pool. The parameters used in this model and throughout the study are as follows: $\lambda 01$, fractional rate of cholesterol loss as neutral sterol; $\lambda 31$, fractional rate of cholesterol conversion to cholate; $\lambda 51$, fractional rate of cholesterol conversion to chenodeoxycholate; $\lambda 21$, fractional rate of transfer of RMP cholesterol to the SMP; $\lambda 12$, fractional rate of transfer of SMP cholesterol to the RMP; $\lambda 43$, fractional rate of cholate conversion to deoxycholate; $\lambda 03$, fractional rate of cholate loss; $\lambda 04$, fractional rate of deoxycholate loss; $\lambda 05$, fractional rate of chenodeoxycholate loss. The $\rho$ symbol indicates the steady-state transport rates in $\mathrm{mg} /$ day for these processes. $M$ represents the size of the pools in mg. $U_{1}$ and $U_{2}$ indicates the steady-state inputs in compartments 1 and 2 .

sterol specific activity declined more rapidly (Fig. 2) than did total plasma cholesterol. After the first day the specific activities of biliary neutral sterol were very close to that of plasma-free cholesterol, indicating a rapid equilibrium between the two. In all six normal studies the biliary neutral sterol specific activity function intersected both the cholate and the chenodeoxycholate functions at the maxima of the latter (Fig. 3). The intersection with cholate always occurred at higher specific activities and at earlier times than that with chenodeoxycholate. The cholate maxima occurred either at the 2 nd or 3rd day, and the chenodeoxycholate maxima was observed between the 4th and 7th day. When considered in light of the precursor product differential equation (17) and the common precursor of both primary bile acids this indicates a more rapid turnover for cholate than cheno- 


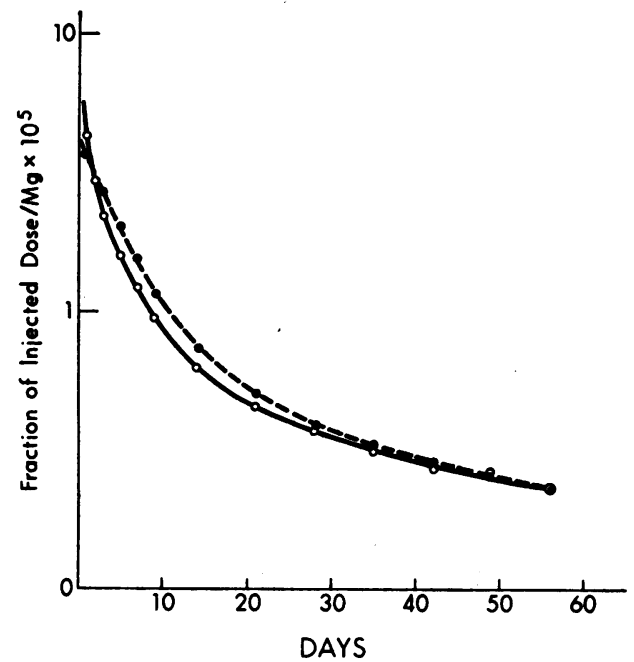

FIGURE 2 The specific activity time course of biliary cholesterol $\bigcirc-\bigcirc$ and total plasma cholesterol -..from subject $E$. Y.

deoxycholate. The cholate specific activity function was also observed to intersect the deoxycholate function at its maximal activity (Fig. 4), usually between the 7th and 10th day of the study. After the intravenous injection of either ${ }^{14} \mathrm{C}$-labeled unconjugated cholic acid or $\left[{ }^{14} \mathrm{C}\right]$ glycocholate the biliary total cholate activity appeared to decay as a single exponential function (Fig. 5).

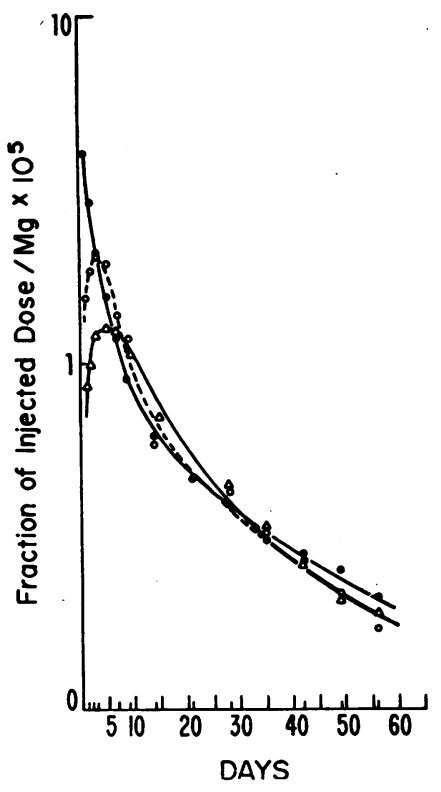

FIgURE 3 The specific activity time course of biliary cholesterol - - , cholic acid $\bigcirc--\bigcirc$, and chenodeoxycholic acid $\triangle \longrightarrow \triangle$ from subject $E$. Y. The points indicate the experimental data and the lines the computer simulation.

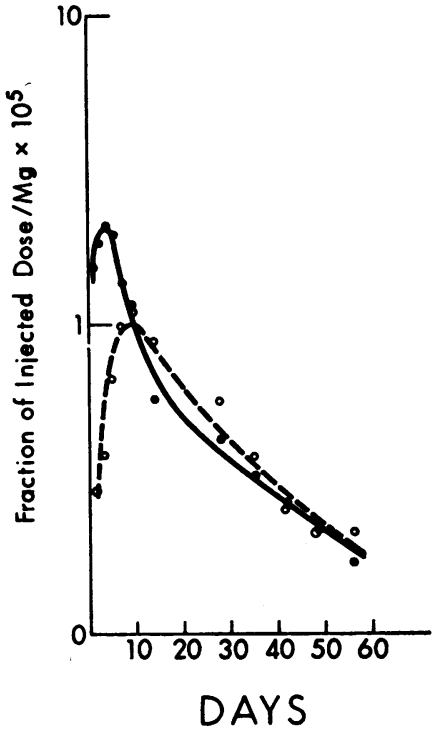

FIGURE 4 The specific activity time course of biliary cholic acid - and deoxycholic acid $\mathrm{O}-\mathrm{C}_{-} \mathrm{O}$ from subject $E$. Y. The points indicate the experimental data and the lines the computer simulations.

The parameters calculated from the compartmental analysis of cholesterol and bile acid kinetics depend to some extent on whether the biliary or plasma cholesterol data are used to represent RMP cholesterol. Differences between the parameters calculated from the two sets of cholesterol data were qualitatively similar for each study and are indicated in Table II. The total $(\lambda 21+\lambda 01+\lambda 31$ $+\lambda 51)$ and irreversible $(\lambda 01+\lambda 31+\lambda 51)$ turnover of RMP cholesterol was greater when biliary neutral sterol data were used. Cholesterol loss as bile acid accounted for somewhat less of the total cholesterol turnover and flux when the calculations were performed with the biliary cholesterol activities. Despite the somewhat slower turnovers of RMP cholesterol when the plasma data were

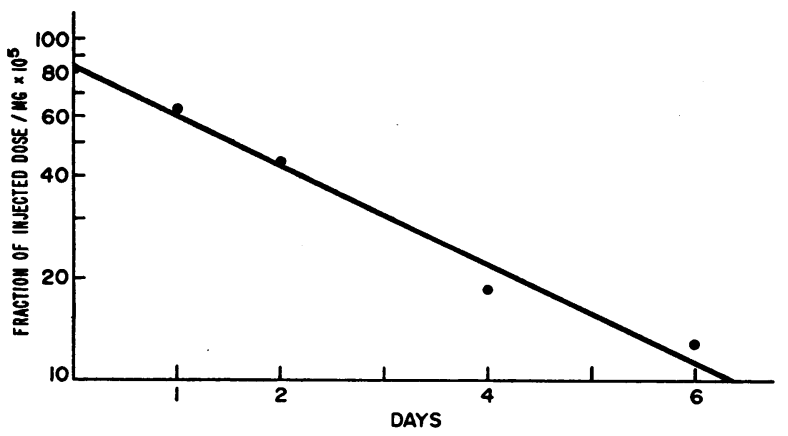

FIgURE 5 The specific activity time course of total biliary cholic acid after the intravenous injection of $\left[24-{ }^{14} \mathrm{C}\right] \mathrm{Na}$ glycocholate in subject $\mathrm{E}$. $\mathrm{Y}$. The line is the regression line obtained from a least squares fit of the data points. 
used the larger RMP pool size yielded a total cholesterol flux $(\rho 01+\rho 31+\rho 51)$ that was nearly identical to that derived with the biliary cholesterol data. Primarily because of the better simulations of the bile acid data achieved from the biliary cholesterol results, the kinetic parameters presented for these subjects are those where biliary cholesterol sequential activities were used for the RMP.

Many of the parameters of the normals in the control state (Table III) were quite similar. The fractional conversion rates of RMP cholesterol to cholic acid ( $\lambda 31$ ) were all about $3 \%$ per day. The fractional conversion rates of chenodeoxycholate were all either $1 \%$ or less each day. The fractional rate of loss of RMP as neutral sterol ( $\lambda 01)$ differed for each of the normals, ranging from $3 \%$ per day in the lean normal, to $9 \%$ per day in one of the obese subjects. The turnover of bile acid was similar for each of the normals. The trihydroxy bile acid catabolic rate was about twice that of the dihydroxy acids. The fractional catabolic rates of biliary cholate obtained from the $\left[{ }^{3} \mathrm{H}\right]$ cholesterol studies were similar to those obtained from the $\left.\mathrm{L}^{-\mathrm{is}} \mathrm{C}\right]$ cholic acid injection in the three normal subjects.

The steady-state calculations in these studies were limited to some extent by the relatively brief duration of the studies. When the plasma cholesterol activities were followed for $50 \mathrm{wk}$ an additional exponential has been described (18) in some humans reflecting a more slowly equilibrating cholesterol pool. The lack of prolonged data in these studies makes it impossible to determine the cholesterol mass in the slowly miscible pool. The total cholesterol turnover and flux may also be overestimated by as much as $14 \%$ (19) if this late appearing exponential is neglected in the calculations. Steady-state results (Table IV) from these relatively short term studies reveal the fluxes in the biliary system and the biliary pool sizes to be relatively similar for all three normals. The conversion of cholate to deoxycholate accounted for between $7-18 \%$ [Table IV, $\rho 43 /(\rho 43+\rho 03)]$ of total cholate metabolism. The largest difference between the three subjects was in their neutral sterol flux ( $\rho 01)$ out of the system.
TABLE II

Cholesterol and Bile Acid Kinetic Parameters of a Normal Derived from the Biliary and Plasma Cholesterol Data*

\begin{tabular}{ccc}
\hline & $\begin{array}{c}\text { Biliary } \\
\text { cholesterol }\end{array}$ & $\begin{array}{c}\text { Plasma } \\
\text { cholesterol }\end{array}$ \\
$\lambda 21 \ddagger$ & $0.23(36) \S$ & $0.13(36)$ \\
$\lambda 01$ & $0.034(43)$ & $0.016(61)$ \\
$\lambda 31$ & $0.026(17)$ & $0.026(18)$ \\
$\lambda 51$ & $0.009(15)$ & $0.009(14)$ \\
$\lambda 43$ & $0.075(18)$ & $0.075(18)$ \\
$\lambda 03$ & $0.35(34)$ & $0.50(32)$ \\
$\lambda 04$ & $0.21(21)$ & $0.22(21)$ \\
$\lambda 05$ & $0.20(22)$ & $0.25(21)$ \\
& & $m g$ or $m g / d a y$ \\
M1 & $15,873(21)$ & $20,661(15)$ \\
M3 & $1,235(16)$ & $1,235(16)$ \\
M4 & $446(24)$ & $446(24)$ \\
M5 & $943(19)$ & $943(19)$ \\
$\rho 01$ & $540(49)$ & $331(64)$ \\
$\rho 31$ & $413(27)$ & $537(24)$ \\
$\rho 51$ & $144(26)$ & $176(21)$ \\
$\rho 43$ & $93(24)$ & $93(24)$ \\
$\rho 03$ & $432(39)$ & $618(36)$ \\
\hline
\end{tabular}

* The parameters presented are those obtained from the normal lean subject $\mathrm{R}$. S.

$\ddagger$ Symbols are described in the legend to Fig. 1.

$\S$ The fractional standard deviations expressed as percent and indicated in the parenthesis.

Two of the normals were fed corn oil, and the kinetic studies were repeated. Despite a fall in plasma cholesterol concentration there was almost no change in the kinetic parameters of one of the subjects (Table V, E. Y.). On the corn oil diet the other normal (Table V, R. S.) demonstrated no significant change in either the total fractional catabolic rate of cholesterol $(\lambda 01+$ $\lambda 31+\lambda 51)$ or total cholesterol transport $(\rho 01+\rho 31+$ $\rho 51$ ). However, the fractional catabolic rates of each of the three bile acids increased as did their fluxes.

One of the obese subjects was treated with cholestyramine, and pronounced change were observed in the

TABLE III

Cholesterol and Bile Acid Fractional Rate Constants in Normals

\begin{tabular}{llcccccccc}
\hline Study & \multicolumn{1}{c}{$\lambda 21^{*}$} & $\lambda 12$ & $\lambda 01$ & $\lambda 31$ & $\lambda 51$ & $\lambda 03$ & $\lambda 05$ & $\lambda 43$ & $\lambda 04$ \\
\hline & & & & & $d a y^{-1}$ & & & & \\
R. S. & $0.23(19) \ddagger$ & $0.11(19)$ & $0.034(43)$ & $0.026(17)$ & $0.009(15)$ & $0.35(34)$ & $0.20(22)$ & $0.075(18)$ & $0.21(21)$ \\
D. H. & $0.15(27)$ & $0.07(20)$ & $0.059(34)$ & $0.038(16)$ & $0.011(14)$ & $0.40(29)$ & $0.20(22)$ & $0.032(15)$ & $0.17(16)$ \\
E. Y. & $0.14(13)$ & $0.06(11)$ & $0.089(34)$ & $0.025(10)$ & $0.004(24)$ & $0.37(17)$ & $0.11(20)$ & $0.051(28)$ & $0.17(25)$ \\
\hline
\end{tabular}

* Kinetic symbols are described in the legend to Fig. 1.

$\ddagger$ The fractional standard deviation is expressed as a percent and indicated within the parenthesis. 
TABle IV

Cholesterol and Bile Acid Pool Sizes and Fluxes in Normals

\begin{tabular}{|c|c|c|c|c|c|c|c|c|c|c|c|c|}
\hline Subject & $\mathbf{M}_{1} *$ & $\mathrm{Mz}_{3}$ & $\mathrm{M}_{4}$ & $M_{6}$ & $\rho 21$ & $p 01$ & $\rho 31$ & $\rho 51$ & $\rho 43$ & $\rho 03$ & $\rho 04$ & p05 \\
\hline & \multicolumn{4}{|c|}{$m g$} & \multicolumn{4}{|c|}{$m g / d a y$} & \multicolumn{4}{|c|}{$m g / d a y$} \\
\hline R. S. & $\begin{array}{c}15,873 \ddagger \\
(21)\end{array}$ & $\begin{array}{r}1,235 \\
(16)\end{array}$ & $\begin{array}{l}446 \\
(24)\end{array}$ & $\begin{array}{l}943 \\
(19)\end{array}$ & $\begin{array}{l}3,651 \\
\quad(29)\end{array}$ & $\begin{array}{l}540 \\
(49)\end{array}$ & $\begin{array}{l}413 \\
(27)\end{array}$ & $\begin{array}{l}144 \\
(26)\end{array}$ & $\begin{array}{c}93 \\
(24)\end{array}$ & $\begin{array}{l}432 \\
(38)\end{array}$ & $\begin{array}{c}94 \\
(32)\end{array}$ & $\begin{array}{l}189 \\
(29)\end{array}$ \\
\hline D. H. & $\begin{array}{c}16,949 \\
(19)\end{array}$ & $\begin{array}{r}1,539 \\
(24)\end{array}$ & $\begin{array}{c}341 \\
(30)\end{array}$ & $\begin{array}{r}1,191 \\
(28)\end{array}$ & $\begin{array}{r}2,542 \\
(33)\end{array}$ & $\begin{array}{l}814 \\
(39)\end{array}$ & $\begin{array}{l}644 \\
(25)\end{array}$ & $\begin{array}{l}186 \\
(24)\end{array}$ & $\begin{array}{c}49 \\
(29)\end{array}$ & $\begin{array}{l}615 \\
(38)\end{array}$ & $\begin{array}{c}58 \\
(34)\end{array}$ & $\begin{array}{l}238 \\
(36)\end{array}$ \\
\hline E. Y. & $\begin{array}{c}21,277 \\
(11)\end{array}$ & $\begin{array}{c}1,235 \\
(20)\end{array}$ & $\begin{array}{l}457 \\
(27)\end{array}$ & $\begin{array}{l}917 \\
(22)\end{array}$ & $\begin{array}{r}2,979 \\
(17)\end{array}$ & $\begin{array}{c}1,894 \\
(36)\end{array}$ & $\begin{array}{l}532 \\
(15)\end{array}$ & $\begin{array}{c}85 \\
(27)\end{array}$ & $\begin{array}{c}63 \\
(35)\end{array}$ & $\begin{array}{l}457 \\
(26)\end{array}$ & $\begin{array}{c}78 \\
(37)\end{array}$ & $\begin{array}{l}101 \\
(30)\end{array}$ \\
\hline
\end{tabular}

* The symbols are described in the legend to Fig. 1.

$¥$ The fractional standard deviation is expressed as a percent and indicated in the parenthesis.

kinetic parameters (Table VI). About a twofold increase was seen in the daily fractional conversion of RMP cholesterol to cholic and chenodeoxycholic acid. A corresponding increase was observed for the flux of cholesterol to cholate and chenodeoxycholate. A higher fraction of cholate transport went to deoxycholate after treatment with cholestyramine [Table VI, $\rho 43 /(\rho 43+$ $\rho 03)]$. Although total transport out of the system increased as a result of Cholestyramine treatment, the neutral sterol fractional catabolic rate and flux appeared to decrease. The RMP size did not diminish with this treatment, but a shift was noted in the relative pool sizes of the three major bile acids.

TABLE V

Cholesterol and Bile Acid Kinetic Parameters in Normals on a Polyunsaturated Diet*

\begin{tabular}{|c|c|c|c|c|}
\hline & \multicolumn{2}{|c|}{ R. S. } & \multicolumn{2}{|c|}{ E. Y. } \\
\hline & Control & Corn oil & Control & Corn oil \\
\hline & \multicolumn{4}{|c|}{$d a y^{-1}$} \\
\hline$\lambda 01 \dagger$ & $0.034(43) \S$ & $0.011(139)$ & $0.089(34)$ & $0.082(20)$ \\
\hline$\lambda 31$ & $0.026(17)$ & $0.031(19)$ & $0.025(10)$ & $0.026(12)$ \\
\hline$\lambda 51$ & $0.009(15)$ & $0.024(22)$ & $0.004(24)$ & $0.008(24)$ \\
\hline$\lambda 43$ & $0.075(18)$ & $0.17(24)$ & $0.051(28)$ & $0.044(11)$ \\
\hline$\lambda 03$ & $0.35(34)$ & $0.54(42)$ & $0.37(17)$ & $0.39(21)$ \\
\hline$\lambda 04$ & $0.21(21)$ & $0.59(26)$ & $0.17(25)$ & $0.14(12)$ \\
\hline \multirow[t]{2}{*}{$\lambda 05$} & $0.20(22)$ & $0.82(32)$ & $0.11(20)$ & $0.19(14)$ \\
\hline & \multicolumn{4}{|c|}{$m g$ or $m g / d a y$} \\
\hline M1 & $15,873(21)$ & $21,739(17)$ & $21,277(11)$ & $19,231(17)$ \\
\hline M3 & $1,235(16)$ & $1,205(15)$ & $1,235(20)$ & $1,299(19)$ \\
\hline M4 & $446(24)$ & $437(33)$ & $457(21)$ & $470(25)$ \\
\hline M5 & $943(19)$ & $917(20)$ & $917(22)$ & $1,000(21)$ \\
\hline$\rho 01$ & $540(49)$ & $239(142)$ & $1,894(36)$ & $1,577(23)$ \\
\hline$\rho 31$ & $413(27)$ & $674(26)$ & $532(15)$ & $500(17)$ \\
\hline$\rho 51$ & $144(26)$ & $522(22)$ & $85(27)$ & $154(27)$ \\
\hline$\rho 43$ & $93(24)$ & $205(29)$ & $63(35)$ & $57(22)$ \\
\hline$\rho 03$ & $432(38)$ & $651(45)$ & $457(26)$ & $507(29)$ \\
\hline
\end{tabular}

* Both patients were on the corn oil diet described in the text. Plasma cholesterol concentrations on the control and corn oil diets are indicated in Table I.

† Symbols described in legend to Fig. 1.

$\$$ The fractional standard deviation is expressed as a percent and indicated in the parenthesis.
The cirrhotic patient demonstrated a number of major differences in his cholesterol and bile acid kinetics ( $\mathrm{Ta}$ ble VII). No deoxycholate could be identified in the bile of this cirrhotic individual. The fractional loss of cholesterol as neutral sterol and the fractional conversion to cholic acid were substantially less than the normals. However, because of the much larger size of the rapidly miscible cholesterol pool, the daily neutral sterol and bile acid fluxes were close to normal. The lower synthetic rate of cholic acid and the accelerated fractional catabolism of this bile acid resulted in a cholic acid pool size for this patient that was significantly less than normal. Although the fractional catabolic rate of chenodeoxycholate was much less well determined, it also appeared to be greater than normal.

\section{DISCUSSION}

The evaluation of human cholesterol kinetics by sequential determinations of biliary cholesterol and bile acid specific activities permits an estimate of both the neutral sterol and bile acid turnover. The analysis of this data does not require the assumption that all cholesterol catabolism occurs from the RMP. However, in these seven studies, both the primary bile acid curves were compatible with an origin solely from the RMP, and since neutral sterol loss occurs mainly by way of biliary cholesterol, this assumption appears to be justified.

The biliary cholesterol specific activity time course had the expected precursor-product relationship to both of the primary bile acids. This relationship is also apparent for the cholate and deoxycholate curves. The maxima for the bile acid specific activity curve occurred either at day 2 or 3 for cholate, between days 4 and 7 for chenodeoxycholate, and between days 7 and 10 for deoxycholate. These values are somewhat earlier than those described by Lindstedt (20) for the cholate and deoxycholate peaks of two normals. The labeled cholesterol was administered orally in his studies, a factor that may account for some delay in the biliary bile acid 
maxima. The cholic specific activity curves of two hyperlipemic patients (21) peaked at 10 days indicating a slower cholate metabolism either caused by the metabolic disorder or induced by the synthetic diet.

The kinetic parameters obtained using the biliary lipid data differed from the results of compartmental analysis of total plasma cholesterol (6). The more rapid total and irreversible turnover of RMP cholesterol and the smaller size of this pool can be attributed to the use of the biliary cholesterol data for this analysis. The $1.1 \mathrm{~g}$ total daily cholesterol loss of the lean subjects (R. S.) was similar to that previously determined from plasma total cholesterol data for people of ideal body weight (8). The two obese subjects were heavier than any of the subjects described by Nestel, Whyte, and Goodman (8), and had daily cholesterol losses somewhat larger than this group. About one-half of the cholesterol flux occurred as neutral sterol for two of the subjects. The obese female (E. Y.), with the largest daily loss, appeared to lose most of her cholesterol as neutral sterol. If the increased cholesterol flux which has been demonstrated to accompany obesity (8) is largely neutral sterol, as in this individual, it may in part explain the predis-

TABLE VI

Cholestyramine Alterations of Cholesterol and Bile Acid Kinetics*

\begin{tabular}{|c|c|c|}
\hline & \multicolumn{2}{|c|}{ D. $\mathrm{H}$. } \\
\hline & Control & Cholestyramine \\
\hline \multicolumn{3}{|c|}{$d a y^{-1}$} \\
\hline$\lambda 01 \ddagger$ & $0.059(34) \S$ & $0.028(38)$ \\
\hline$\lambda 31$ & $0.038(16)$ & $0.078(12)$ \\
\hline$\lambda 51$ & $0.011(14)$ & $0.019(14)$ \\
\hline$\lambda 43$ & $0.032(15)$ & $0.13(20)$ \\
\hline$\lambda 03$ & $0.40(29)$ & $0.69(20)$ \\
\hline$\lambda 04$ & $0.17(16)$ & $1.8(23)$ \\
\hline$\lambda 05$ & $0.20(22)$ & $0.96(16)$ \\
\hline \multicolumn{3}{|c|}{$m g$ or $m g / d a y$} \\
\hline M1 & $16,949(19)$ & $23,256(12)$ \\
\hline M3 & $1,539(24)$ & $2,273(7)$ \\
\hline M4 & $341(30)$ & $134(46)$ \\
\hline M5 & $1,191(28)$ & $529(25)$ \\
\hline$\rho 01$ & $814(39)$ & $651(40)$ \\
\hline$\rho 31$ & $644(25)$ & $1,814(17)$ \\
\hline$\rho 51$ & $186(24)$ & $442(19)$ \\
\hline$\rho 43$ & $49(29)$ & $296(21)$ \\
\hline$\rho 03$ & $615(38)$ & $1,568(21)$ \\
\hline
\end{tabular}

* Mean plasma cholesterol $\pm \mathrm{SEM}$ in the control and cholestyramine periods are indicated in Table I.

† Symbols described in legend to Fig. 1 .

$\S$ The fractional standard deviation is expressed as a percent and indicated within the parenthesis.
TABLE VII

Cholesterol and Bile Acid Kinetic Parameters of a Cirrhotic*

\begin{tabular}{|c|c|c|}
\hline & Normal (R. S.) & Cirrhosis (D. T.) \\
\hline \multicolumn{3}{|c|}{$d a y^{-1}$} \\
\hline$\lambda 01 \ddagger$ & $0.034(43) \S$ & $0.015(46)$ \\
\hline$\lambda 31$ & $0.026(17)$ & $0.012(13)$ \\
\hline$\lambda 51$ & $0.009(15)$ & $0.003(190)$ \\
\hline$\lambda 43$ & $0.075(18)$ & \\
\hline$\lambda 03$ & $0.35(34)$ & $0.9(18)$ \\
\hline$\lambda 04$ & $0.21(21)$ & \\
\hline$\lambda 05$ & $0.20(22)$ & $0.9(190)$ \\
\hline \multicolumn{3}{|c|}{$m g$ or $m g / d a y$} \\
\hline M1 & $15,873(21)$ & $37,037(8)$ \\
\hline M3 & $1,235(16)$ & $606(8)$ \\
\hline M4 & $446(24)$ & \\
\hline M5 & $943(19)$ & $235(16)$ \\
\hline$\rho 01$ & $540(49)$ & $556(47)$ \\
\hline p31 & $413(27)$ & $444(15)$ \\
\hline$\rho 51$ & $144(26)$ & $111(191)$ \\
\hline$\rho 43$ & $93(24)$ & \\
\hline$\rho 03$ & $432(38)$ & $545(20)$ \\
\hline
\end{tabular}

* A patient with macronodular cirrhosis described in Table I. $\ddagger$ The symbols are described in legend to Fig. 1 .

$\$$ The fractional standard deviation is expressed as a percent and indicated within the parenthesis.

position of some overweight humans to cholesterol gallstone disease.

The normal fractional catabolic rates and transports of the three major bile acids obtained from these labeled cholesterol studies were not greatly different from those obtained from labeled bile acid studies $(22,23)$. It appeared from these three normals that both chenodeoxycholate and deoxycholate were metabolized at half the rate of cholic acid. Less than $20 \%$ of cholate turnover went to deoxycholate in these normals.

The small number of subjects studied does not permit definite conclusions about the effects of either corn oil, cholestyramine, or cirrhosis on cholesterol and bile acid kinetics. The results do indicate, however, that the model is appropriate for the analysis of these perturbations and provides suggestions about their differences from normal cholesterol kinetics. Similar to previous studies of corn oil $(24,25)$ neither of these subjects had a significant increment in cholesterol loss from the body. One subject appeared to, however, redistribute his cholesterol flux, losing less as neutral sterol and more as bile acid.

Cholestyramine, as noted previously (6), produced a significant increment in the total sterol loss from the body. This increase was almost entirely in bile acid flux with no significant change in neutral sterol transport. The dihydroxy bile acid pool sizes decreased to some ex-

Cholesterol and Bile Acid Kinetics in Man

1943 
tent but not in proportion to the large increments in their catabolic rates. Despite an increase in cholate catabolism the pool size of this bile acid appeared to increase. Similar altered bile acid pool sizes have been published for normals (26) after cholestyramine treatment.

The cirrhotic patient demonstrated diminished fractional conversion of cholesterol to cholic acid and loss as neutral sterol, but a larger RMP and resultant fluxes that approximated the normal. The fractional turnover rates for cholic and chenodeoxycholic acids obtained in this cirrhotic were greater than those previously published (27) by a labeled bile acid method. The slower catabolic rates observed in the labeled bile acid studies may in part be explained by the slower uptake of labeled bile acid from the circulation into the liver and the greater reflux from the liver, both of which produce a larger extrahepatic bile acid pool in cirrhotics (28). This alteration in the hepatic clearance of plasma bile acid could have the effect of producing a prolonged infusion of labeled bile acid into the biliary tract, creating a spuriously slow biliary exponential decay.

The analysis of biliary lipid after an intravenous injection of labeled cholesterol provides measures of cholesterol and bile acid metabolism that cannot be obtained by either the previously described plasma or fecal methods alone. The results of such an analysis indicate acidic and neutral sterol fluxes that are not greatly different from previous determinations $(2,3)$ by other methods. Although the present study used a multicompartmental analysis of this data, many of the kinetic parameters are model invariant and can be determined by other mathematical approaches. The method is currently being applied to the study of both hyperlipoproteinemic states and cholesterol gallstone disease.

\section{ACKNOWLEDGMENTS}

The authors would like to thank Miss Helen Hilderman and Mrs. Marie Dowdee for their technical assistance, and Mrs. Sadie S. Goodman for her excellent secretarial assistance.

This work was supported in part by a grant from the National Institutes of Health, 5 RO1 HL14313-02, and a grant from the North Carolina Heart Association, no. 197071-A-2.

\section{REFERENCES}

1. Hellman, L., R. S. Rosenfeld, W. Insull, Jr., and E. H. Ahrens, Jr. 1957. Intestinal excretion of cholesterol : a mechanism for regulation of plasma levels. J. Clin. Invest. $36:$ 898. (Abstr.)

2. Wilson, J. D., and C. A. Lindsey, Jr. 1965. Studies on the influence of dietary cholesterol on cholesterol metabolism in the isotopic steady state in man. J. Clin. Invest. 44: 1805.

3. Grundy, S. M., and E. H. Ahrens, Jr. 1966. An evaluation of the relative merits of two methods for measur- ing the balance of sterols in man: isotopic balance versus chromatographic analysis. J. Clin. Invest. 45: 1503.

4. Grundy, S. M., and E. H. Ahrens, Jr. 1969. Measurement of cholesterol turnover, synthesis, and absorption in man, carried out by isotope kinetic and sterol balance methods. J. Lipid Res. 10: 91.

5. Chobanian, A. V., B. A. Burrows, and W. Hollander. 1962. Body cholesterol metabolism in man. II. Measurement of the body cholesterol miscible pool and turnover rate. J. Clin. Invest. 41: 1738.

6. Goodman, DeW. S., and R. P. Noble. 1968. The turnover of plasma cholesterol in man. J. Clin. Invest. 47: 231.

7. Samuel, P., C. M. Holtzman, E. Meilman, and W. Perl. 1968. Effect of Neomycin on exchangeable pools of cholesterol in the steady state. J. Clin. Invest. 47: 1806.

8. Nestel, P. J., H. M. Whyte, and D. S. Goodman. 1969. Distribution and turnover of cholesterol in humans. $J$. Clin. Invest. 48: 982.

9. Austad, W. I., L. Lack, and M. P. Tyor. 1967. Importance of bile acids and of an intact distal small intestine for fat absorption. Gastroenterology. 52: 638 .

10. Abell, L. L., B. B. Levy, B. B. Brodie, and F. E. Kendall. 1952. Simplified method for estimation of total cholesterol in serum and demonstration of its specificity. J. Biol. Chem. $195: 357$.

11. Dole, V. P. 1956. A relation between non-esterified fatty acids in plasma and the metabolism of glucose. J. Clin. Invest. $35: 150$.

12. Miettinen, T. A., E. H. Ahrens, Jr., and S. M. Grundy. 1965. Quantitative isolation and gas-liquid chromatographic analysis of total dietary and fecal neutral steroids. J. Lipid Res. 6: 411.

13. Grundy, S. M., E. H. Ahrens, Jr., and T. A. Miettinen. 1965. Quantitative isolation and gas-liquid chromatographic analysis of total fecal bile acids. J. Lipid Res. 6: 397.

14. Talalay, P. 1962. Hydroxysteroid dehydrogenases. Methods Enzymol. 5 : 512.

15. Sandberg, D. H., J. Sjövall, K. Sjövall, and D. A Turner. 1965. Measurement of human serum bile acids by gas-liquid chromatography. J. Lipid Res. 6: 182.

16. Berman, M., and M. F. Weiss. 1967. SAAM Manual. U. S. Department of Health, Education and Welfare, Public Health Service, U. S. Government Printing Office, Washington, D. C.

17. Zilversmit, D. B. 1960. The design and analysis of isotope experiments. Review. Am. J. Med. 29: 832.

18. Samuel, P., and W. Perl. 1970. Long term decay of serum cholesterol radioactivity: body cholesterol metabolism in normals and in patients with hyperlipoproteinemia and atherosclerosis. J. Clin. Invest. 49: 346.

19. Goodman, D. S., R. P. Noble, and R. B. Dell. 1972. Three-pool model of long term turnover of plasma cholesterol in man. Circulation. 45 : II-249.

20. Lindstedt, S. 1962. Equilibration of dietary cholesterol and bile acids in man. Bile acids and steroids. Clin. Chim. Acta. $7: 1$.

21. Lindstedt, S., and E. H. Ahrens, Jr. 1961. Conversion of cholesterol to bile acids in man. Proc. Soc. Exp. Biol. Med. 108 : 286.

22. Hepner, G. W., A. F. Hofmann, and P. J. Thomas. 1972. Metabolism of steroid and amino acid moieties of conjugated bile acids in man. J. Clin. Invest. 51: 1898. 
23. Vlahcevic, Z. R., J. R. Miller, J. T. Farrar, and L. Swell. 1971. Kinetics and pool size of primary bile acids in man. Gastroenterology. $61: 85$.

24. Avigan, J., and D. Steinberg. 1965. Sterol and bile acid excretion in man and the effects of dietary fat. J. Clin. Invest. $44: 1845$.

25. Spritz, N., E. H. Ahrens, Jr., and S. Grundy. 1965 Sterol balance in man as plasma cholesterol concentrations are altered by exchanges of dietary fats. J. Clin. Invest. $44: 1482$.
26. Garbutt, J. T., and T. J. Kenney. 1972. Effect of Cholestyramine on bile acid metabolism in normal man. J. Clin. Invest. $51: 2781$

27. Vlahcevic, Z. R., I. Buhac, J. T. Farrar, C. C. Bell, Jr., and L. Swell. 1971. Bile acid metabolism in patients with cirrhosis. I. Kinetic aspects of cholic acid metabolism. Gastroenterology. 60: 491.

28. Blum, M., and N. Spritz. 1966. The metabolism of intravenously injected isotopic cholic acid in Laennec's cirrhosis. J. Clin. Invest. 45: 187. 